\title{
Comparison of stapler and electrocautery for division of the intersegmental plane in lung segmentectomy
}

\author{
Tomohiro Yazawa, Hitoshi Igai^, Kazuki Numajiri, Fumi Ohsawa, Natsumi Matsuura, \\ Mitsuhiro Kamiyoshihara
}

Department of General Thoracic Surgery, Japanese Red Cross Maebashi Hospital, Maebashi, Japan

Contributions: (I) Conception and design: T Yazawa, H Igai; (II) Administrative support: T Yazawa, H Igai; (III) Provision of study materials or patients: All authors; (IV) Collection and assembly of data: All authors; (V) Data analysis and interpretation: T Yazawa, H Igai; (VI) Manuscript writing: All authors; (VII) Final approval of manuscript: All authors.

Correspondence to: Hitoshi Igai, MD, PhD. Department of General Thoracic Surgery, Japanese Red Cross Maebashi Hospital, 389-1, Asakura, Maebashi, 371-0811 Gunma, Japan. Email: hitoshiigai@gmail.com.

Background: We retrospectively compared the use of a stapler and electrocautery for division of the intersegmental plane during pulmonary segmentectomy.

Methods: We enrolled 156 patients who underwent pulmonary segmentectomy in our department between March 2006 and August 2020. The patients were divided into electrocautery $(n=62)$ and stapler $(n=94)$ groups based on the device used to divide the intersegmental plane. Patient characteristics, perioperative outcomes, and ratios of actual (calculated using software) to predicted (calculated by counting the resected segments) lung volumes were compared between the two groups. Additionally, we used multivariate analysis to identify the factors that contributed to the incidence of postoperative air leakage after cut-off value was set by receiver operating characteristic (ROC) curve analysis. Moreover, a subset analysis was performed based on the type of segmentectomy (common or uncommon). Common segmentectomies included resection of the basilar or superior segment of the lower lobe, or lingular or upper division of the left upper lobe; all other segmentectomies were classified as uncommon.

Results: Compared to the electrocautery group, the stapler group had shorter operative times $(\mathrm{P}=0.0027)$, duration of postoperative drainage $(\mathrm{P}=0.00037)$, and duration of postoperative hospitalization $(\mathrm{P}=0.0021)$. Moreover, incidence of postoperative $\geq 3$ days drainage was significantly reduced in the stapler group $(\mathrm{P}=0.003)$. There were no significant differences between the stapler and electrocautery groups in the actual:predicted lung volumes at 6 months (1.01 and 1.04, respectively; $\mathrm{P}=0.28)$ or 12 months (1.06 and 1.07, respectively; $\mathrm{P}=0.68)$ after surgery. Preoperative lung volume was significantly correlated with preoperative vital capacity $(\mathrm{VC})(\gamma=0.69 ; \mathrm{P}<0.001)$ and forced expiratory volume in 1 second $\left(\mathrm{FEV}_{1}\right)(\gamma=0.48 ; \mathrm{P}<0.001)$. The multivariate analysis indicated that the use of stapler for division of intersegmental plane was the only factor that contributed to reducing the incidence of postoperative $\geq 3$ days drainage $(\mathrm{P}=0.0027$, odds ratio: 0.23, 95\% CI: $0.086-0.597)$. In a subset analysis of uncommon segmentectomy, there were no significant differences among the groups in most perioperative results.

Conclusions: Compared to electrocautery, the use of a stapler for division of the intersegmental plane was associated with better perioperative outcomes, especially reduction of postoperative drainage time, and similar postoperative remnant lung volumes and function.

Keywords: Segmentectomy; intersegmental plane; stapler; electrocautery

Submitted Aug 23, 2021. Accepted for publication Oct 14, 2021.

doi: $10.21037 /$ jtd-21-1397

View this article at: https://dx.doi.org/10.21037/jtd-21-1397

$\wedge$ ORCID: 0000-0002-5916-8209.

(C) Journal of Thoracic Disease. All rights reserved. 


\section{Introduction}

Lobectomy is the standard surgical procedure for primary lung cancer. However, several studies have demonstrated superior perioperative results and cancer outcomes after pulmonary segmentectomy compared to lobectomy (1-3). Therefore, pulmonary segmentectomy is increasingly being performed worldwide.

Pulmonary segmentectomy offers the advantage of sparing the lung parenchyma, leading to better lung function. However, the most appropriate method to identify the intersegmental plane during pulmonary segmentectomy is yet to be established. Researchers have studied inflationdeflation techniques, selective inflation of the target segment by jet ventilation, intravenous and endobronchial indocyanine green injection, and virtual-assisted lung mapping for identification of the intersegmental planes (4-8). The best method to divide the intersegmental plane is also controversial; the most commonly used methods are stapling and electrocautery (9-12).

Although the advantages of both techniques are welldocumented, few studies have compared them (13-16). To determine the optimal method for division of the intersegmental plane during pulmonary segmentectomy, we retrospectively compared the use of a stapler and electrocautery in terms of the perioperative outcomes and residual lung volumes.

We present the following article in accordance with the STROBE reporting checklist (available at https://dx.doi. org/10.21037/jtd-21-1397).

\section{Methods}

This study was conducted in accordance with the Declaration of Helsinki (2013 revision), and approved by the institutional ethics board of Japanese Red Cross Maebashi Hospital (No.: 2020-53, date: March 1, 2021). Informed consent was waived for this retrospective analysis.

We reviewed the records of 176 patients who underwent thoracoscopic anatomical pulmonary segmentectomy between March 2006 and August 2020 in our department. Patients who underwent pulmonary resection, radiotherapy or chemotherapy before or after segmentectomy were excluded from this study. Twenty patients were excluded due to incomplete data $(n=3)$, no follow-up computed tomography (CT) scan due to death $(\mathrm{n}=3)$, short-duration follow-up $(n=5)$, transfer to another hospital $(n=5)$, or loss to follow-up $(\mathrm{n}=4)$. Thus, we included 156 patients in this study (Figure 1), and divided them into electrocautery ( $\mathrm{n}=62)$ and stapler $(\mathrm{n}=94)$ groups based on the device used to divide the intersegmental plane. All surgeries were performed by the same team of thoracic surgeons. Patient data obtained from the medical records included age, sex, smoking history, preoperative vital capacity (VC), forced expiratory volume in 1 second $\left(\mathrm{FEV}_{1}\right)$, type of segmentectomy (intentional or unintentional, and common or uncommon), type of tumor (primary, metastatic, or benign), surgical approach (minithoracotomy, multiport thoracoscopic, or uniport thoracoscopic), resected segments, operative time, blood loss, incidence of massive intraoperative bleeding, conversion to thoracotomy, duration of postoperative drainage, duration of postoperative hospitalization, postoperative morbidity (Clavien-Dindo Classification grade $\geq$ III), and readmission within 30 days postoperatively. In addition, we conducted receiver operating characteristic (ROC) curve analysis with the device used to divide the intersegmental plane as the dependent variable and the duration of postoperative drainage as the independent variable to set cut-off values. We compared the patient characteristics and perioperative outcomes between the two groups. In addition, we used multivariate analysis to identify the factors that contributed to the incidence of postoperative air leakage (the cut-off value would be set on the ROC curve analysis) among the parameters in patient's characteristics and the device for division of intersegmental plane. Moreover, a subset analysis was performed based on the type of segmentectomy. Common segmentectomies included resection of the basilar or superior segment of the lower lobe, or lingular or upper division of the left upper lobe; all other segmentectomies were classified as uncommon (17).

\section{Surgical indications}

In our department, intentional pulmonary segmentectomy was performed for the patient with Stage 0 or IA1 nonsmall cell lung cancer when the patient accepted it. Unintentional pulmonary segmentectomy was for the patient without intrathoracic lymph node or distant metastasis who was contraindication for lobectomy due to limited pulmonary function or compromised status. We performed pulmonary segmentectomy for pulmonary metastases when they were completely resectable and too deeply located to perform wedge resection. Finally, all of the benign diseases in this study were initially suspected as malignancy. 


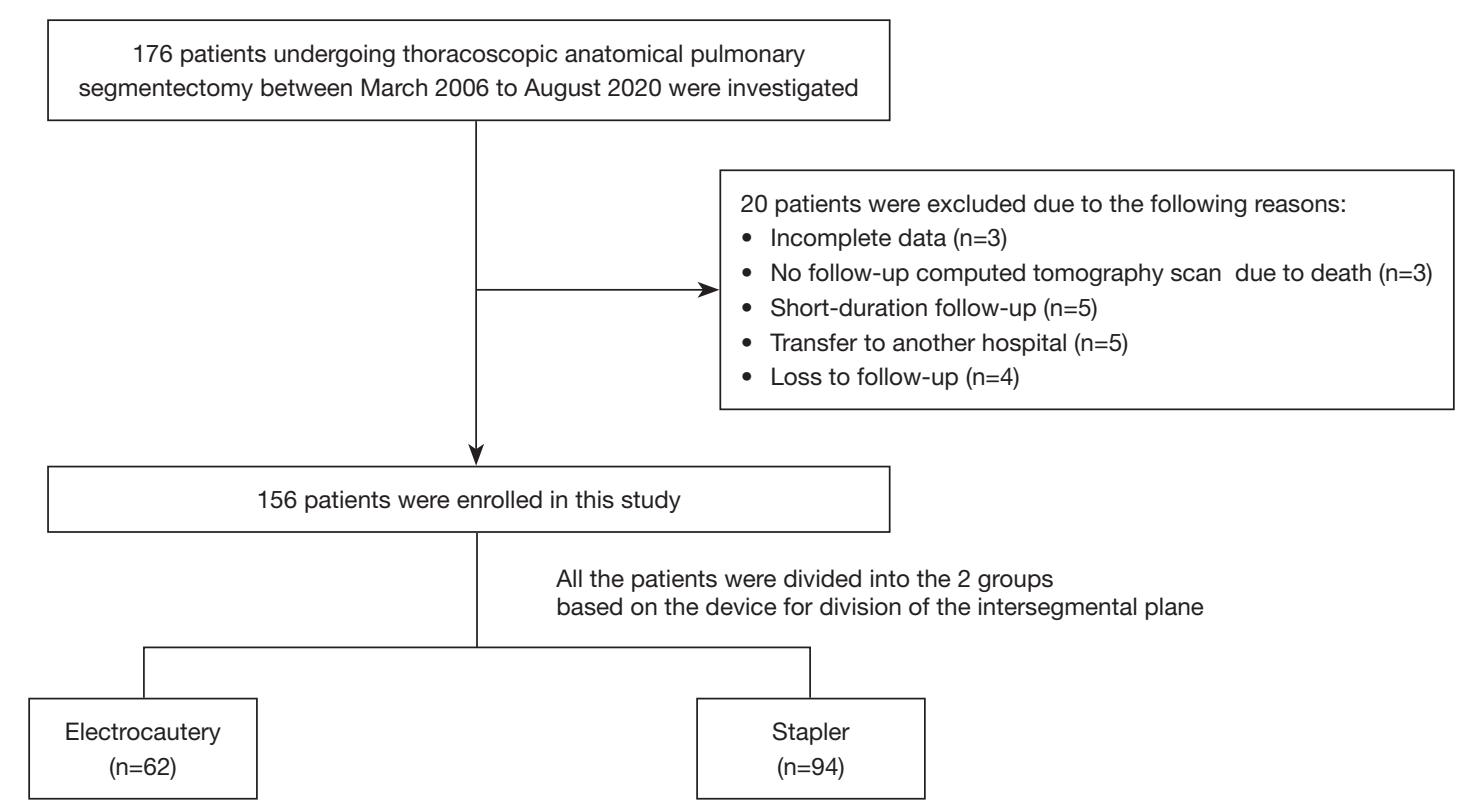

Figure 1 Patient enrollment in this retrospective study.

\section{Surgical procedures}

The surgeries were performed using one-lung ventilation under general anesthesia with the patient in the lateral decubitus position. Different surgical approaches were used: minithoracotomy (from March 2006 to April 2012), a multiportal thoracoscopic approach (from May 2012 to January 2019) and a uniportal or multiportal thoracoscopic approach (from February 2019 to October 2019) and a uniportal thoracoscopic approach (since November 2019). Four surgeons (including HI, MK, NM, or other surgeon) performed any surgeries during the study period. Apart from the differences in approaches, all other surgical steps were similar. The dominant vessels (i.e., pulmonary artery or vein) were divided, and an intersegmental plane was identified using either the conventional inflationdeflation technique or near-infrared imaging after intravenous indocyanine green (ICG) administration (4-6). Intersegmental pulmonary veins in the hilum were used as landmarks to identify the intersegmental plane when an intersegmental plane was not clearly identified using those techniques. Following division of the target segmental bronchus during minithoracotomy or multiportal thoracoscopic approach, the intersegmental plane was divided using electrocautery (monopolar cautery) or stapling. In the minithoracotomy approach, electrocautery was frequently used based on the institutional policy during the period. The choice of the device including electrocautery or staples depended on the surgeons' preference in multiportal approach. Finally, in the uniportal thoracoscopic approach, staples were generally used except for only a few patients because of difficulty in achieving the countertraction necessary for electrocautery. In the stapler group, the intersegmental plane was divided completely using staples. However, at least, two-thirds of outer area in an intersegmental plane was divided by electrocautery for any patients in the electrocautery group. Rest of the deep parenchyma was divided by staples. When we found major air leak, we sutured the leak point with an absorbable string, which was performed in both groups. Subsequently, the leak point was covered with fibrin glue (Bolheal; KM Biologics, Kumamoto, Japan; or Beriplast P; CSL Behring, King of Prussia, PA, USA), and an absorbable polyglycolic acid felt (Neoveil; Gunze Ltd., Kyoto, Japan). In the electrocautery group, the coverage was performed for the divided intersegmental plane in any patients to prevent delayed air leak even if we did not detect air leak in a seal test. Finally, a 24-Fr double-lumen chest tube was placed in the pleural cavity. At the morning round, our team checked the postoperative air leakage and pleural drainage volume. The chest tube was removed after the air leakage had stopped, and the volume of pleural effusion was reduced to $\leq 300 \mathrm{~mL}$ within 24 hours and the lung had expanded, as seen on chest X-ray. When postoperative air leak continued 
for 2 days or longer, we took performing pleurodesis into consideration. In pleurodesis, materials involving OK-432 (Picibanil, Chugai: Pharmaceutical Co. Ltd., Tokyo, Japan), minocycline, or auto-blood was administered into thoracic cavity via the chest tube.

\section{Calculation of the lung volume}

In our department, a 64-row multidetector CT scan was performed for all patients undergoing anatomical pulmonary resection within 2 weeks before surgery, and 6 and 12 months after surgery. The data were imported into Ziostation2 software (Ziosoft, Tokyo, Japan), and reconstructed to three-dimensional CT to calculate the pre- and post-operative lung volumes. Unfortunately, ipsilateral lung volumes during surgery cannot be calculated using this software. Therefore, we evaluated the changes in lung volume based on the pre- and postoperative values. Subsequently, we counted the resected lobes and calculated the postoperative predicted lung volume using the following formula: postoperative predicted lung volume $=$ preoperative lung volume $\times(19$ $-n) / 19$, where $n$ is the number of removed segments (18). The ratios of postoperative actual (calculated by Ziostation 2) and predicted (calculated by the formula above) lung volumes in the stapler and electrocautery groups were compared. Subset analysis was performed based on the type of segmentectomy (i.e., common or uncommon).

In addition, we evaluated the correlations between preoperative lung volume and pulmonary functions (VC and $\left.\mathrm{FEV}_{1}\right)$. Unfortunately, no postoperative lung function was measured in our department.

\section{Statistical analysis}

Fisher's exact test was used to analyze the associations between categorical variables. Moreover, we used ROC curve analysis to set cut-off values for continuous variables. Student's $t$-test was used to analyze continuous variables, including the actual:predicted lung volume ratio. The correlations between variables were analyzed using Pearson's correlation. Multivariate analyses for categorical variables were performed using a logistic regression model. Results were considered significant at $\mathrm{P}<0.05$. Calculations and statistical tests were performed using the EZR graphical user interface (Saitama Medical Centre, Jichi Medical University, Saitama, Japan).

\section{Results}

Table 1 summarizes the preoperative patient characteristics. There were no significant differences between the groups in age, sex, or preoperative pulmonary functions (VC and FEV1). The stapler group included more patients with smoking history than the electrocautery group $(\mathrm{P}=0.0015)$. Intentional or uncommon types of segmentectomy, or uniportal approach were performed more frequently in the stapler than electrocautery group $(\mathrm{P}=0.00022,0.0045,<0.0001)$. The stapler group included more patients with primary lung cancer than the electrocautery group $(\mathrm{P}=0.00031)$.

The details of surgical procedures performed in the electrocautery and stapler groups are presented in Table 2.

ROC curve analysis using the device used to divide the intersegmental plane as the dependent variable and the duration of postoperative drainage as the independent variable revealed an area under the curve (AUC) of 0.68 [95\% confidence interval (CI): 0.6-0.76] (Figure 2). Based on the result (cut-off value: 2 , specificity: 0.473 , sensitivity: 0.825 ), the cut-off threshold of the duration of postoperative drainage was set on $\leq 2$ and $\geq 3$ days.

Table 3 shows the perioperative outcomes of the two groups. There were no significant differences in blood loss, massive bleeding, conversion to thoracotomy, postoperative morbidity, or readmission within 30 days postoperatively between the groups. However, the stapler group had shorter operative times $(\mathrm{P}=0.0027)$, duration of postoperative drainage $(\mathrm{P}=0.00037)$, incidence of postoperative $\geq 3$ days drainage $(\mathrm{P}=0.003)$ and duration of postoperative hospitalization $(\mathrm{P}=0.0021)$ compared to the electrocautery group. In addition, we did not find any significant differences between the stapler and electrocautery groups in terms of the postoperative actual:predicted lung volumes at 6 months (1.02 and 1.04, respectively; $\mathrm{P}=0.35$ ) and 12 months (1.06 and 1.07 , respectively; $\mathrm{P}=0.78$ ). Figure 3 demonstrates that the preoperative lung volumes calculated by Ziostation 2 were significantly correlated with the preoperative $\mathrm{VC}(\gamma=0.69$; $\mathrm{P}<0.001)$ and FEV1 $(\gamma=0.48 ; \mathrm{P}<0.001)$.

In Table 4, the multivariate analysis indicated that the use of stapler for division of intersegmental plane was the only factor that contributed to reducing the incidence of postoperative $\geq 3$ days drainage ( $\mathrm{P}=0.0027$, odds ratio: 0.23 , 95\% CI: 0.086-0.597).

Table 5 summarizes the results of a subset analysis based on preoperative patient characteristics and perioperative outcomes in the group with common type of segmentectomy. The stapler group had significantly shorter 
Table 1 Preoperative characteristics of the two groups

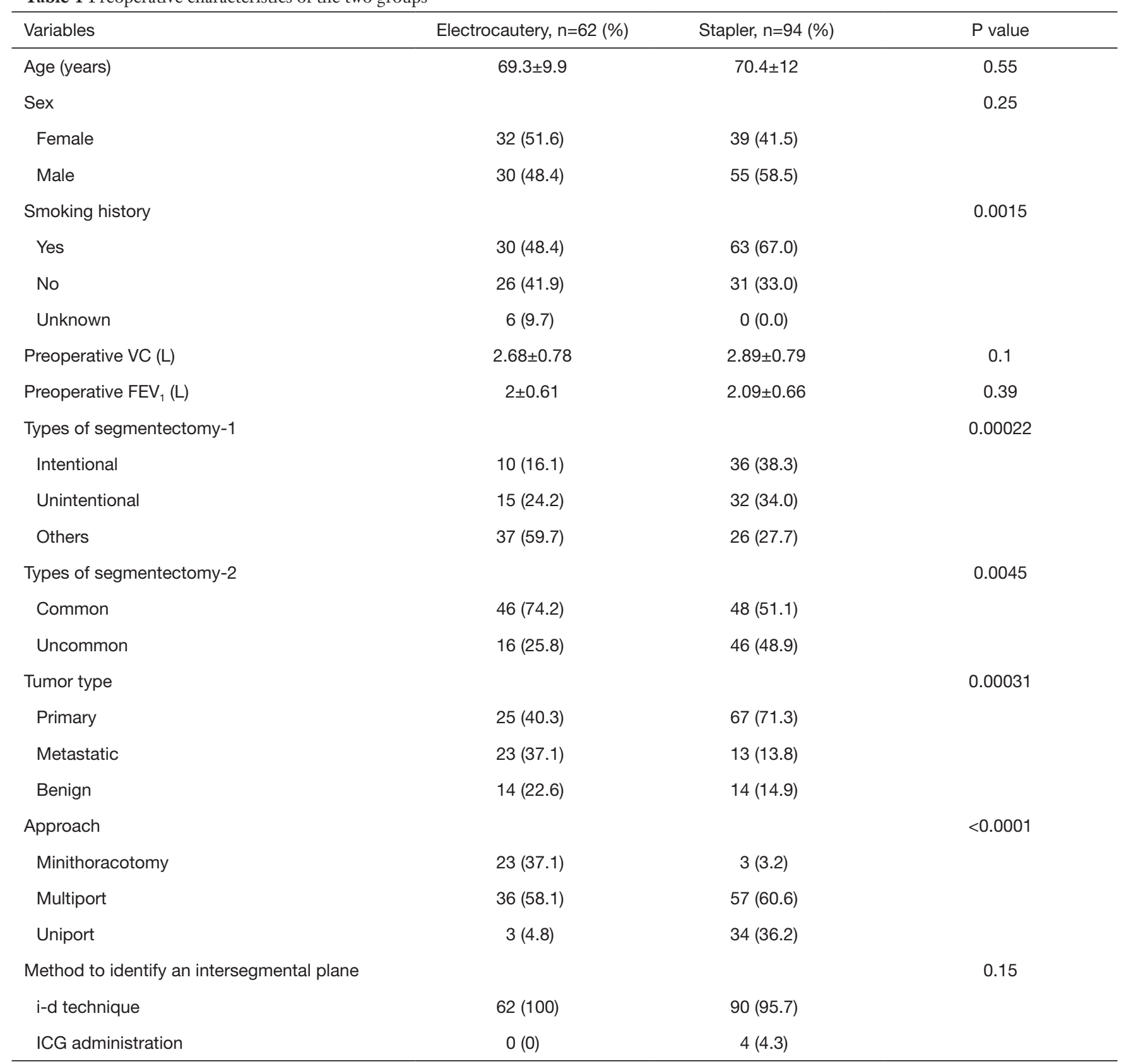

VC, vital capacity; FEV $_{1}$, forced expiratory volume in 1 second; i-d technique, inflation-deflation technique; ICG, indocyanine green.

or less operative times $(\mathrm{P}=0.0025)$, postoperative drainage $(\mathrm{P}=0.0031)$, incidence of postoperative $\geq 3$ days drainage $(\mathrm{P}=0.012)$ and postoperative hospitalization $(\mathrm{P}=0.019)$. Other parameters, including the actual:predicted lung volumes at 6 and 12 months postoperatively, were not significantly different. A subset analysis of the perioperative results of the uncommon segmentectomy group revealed that the stapler group had significantly shorter postoperative hospitalization $(\mathrm{P}=0.022)$ although there were no significant differences about other parameters (Table 6).

\section{Discussion}

In this study, we compared perioperative parameters 
Table 2 Details of surgical procedures performed in the electrocautery and stapler groups

\begin{tabular}{|c|c|c|}
\hline Surgical procedure & $\begin{array}{c}\text { Electrocautery, } \\
n=62\end{array}$ & $\begin{array}{c}\text { Stapler, } \\
\mathrm{n}=94\end{array}$ \\
\hline \multicolumn{3}{|l|}{ LUL } \\
\hline Superior segment: $\mathrm{S}^{1-3}$ & 14 & 13 \\
\hline Lingual segment: $\mathrm{S}^{4-5}$ & 5 & 3 \\
\hline Posterior segment: $\mathrm{S}^{1+2}$ & 3 & 8 \\
\hline Anterior segment: $\mathrm{S}^{3}$ & 2 & 4 \\
\hline Anterior + lingual segment: $S^{3+4+5}$ & 0 & 2 \\
\hline Others & 0 & 2 \\
\hline \multicolumn{3}{|l|}{ LLL } \\
\hline Superior segment: $\mathrm{S}^{6}$ & 7 & 9 \\
\hline Basal segment: $S^{8-10}$ & 5 & 2 \\
\hline Basilar anterior/lateral segment: $\mathrm{S}^{8+9}$ & 0 & 1 \\
\hline Basilar lateral/posterior segment: $\mathrm{S}^{9+10}$ & 1 & 0 \\
\hline \multicolumn{3}{|l|}{ RUL } \\
\hline Apical segment: $S^{1}$ & 2 & 2 \\
\hline Posterior segment: $\mathrm{S}^{2}$ & 1 & 7 \\
\hline Anterior segment: $\mathrm{S}^{3}$ & 3 & 3 \\
\hline Others & 1 & 5 \\
\hline \multicolumn{3}{|l|}{ RLL } \\
\hline Superior segment: $\mathrm{S}^{6}$ & 13 & 14 \\
\hline Basal segment: $S^{7-10}$ & 2 & 7 \\
\hline Basilar medial/anterior segment: $\mathrm{S}^{7+8}$ & 0 & 2 \\
\hline Basilar anterior/lateral segment: $\mathrm{S}^{8+9}$ & 0 & 1 \\
\hline Basilar lateral/posterior segment: $S^{9+10}$ & 3 & 9 \\
\hline
\end{tabular}

LUL, left upper lobe; LLL, left lower lobe; RUL, right upper lobe; $\mathrm{RLL}$, right lower lobe.

and postoperative lung volumes between stapler and electrocautery groups of patients undergoing pulmonary segmentectomy. Compared to electrocautery, stapler use was associated with superior perioperative outcomes, including operative, postoperative drainage, and postoperative hospitalization times. There was also a significant lower incidence of postoperative $\geq 3$ days drainage in the stapler group compared to the electrocautery group. Moreover, the use of stapler for division of intersegmental plane was the only factor that contributed to reducing the incidence of postoperative $\geq 3$ days drainage in the multivariate analysis.

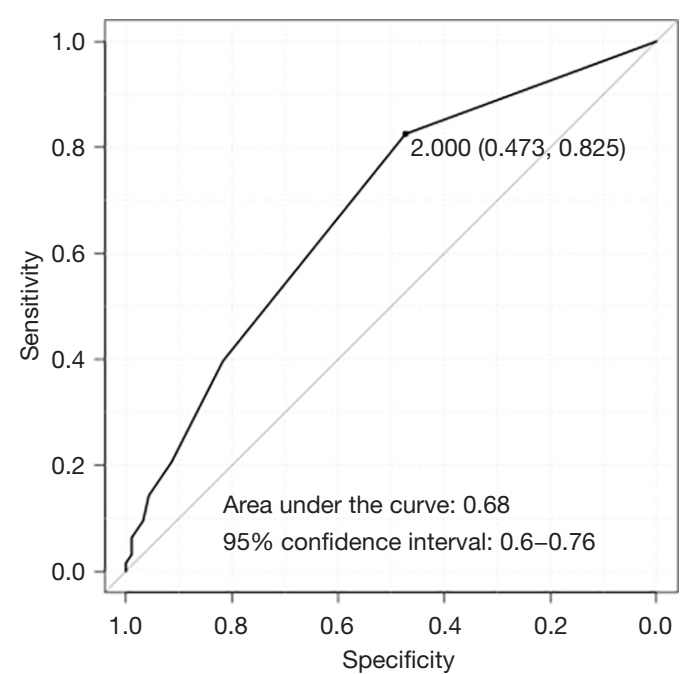

Figure 2 Receiver operating characteristic curve analysis using the device used to divide the intersegmental plane as the dependent variable and the duration of postoperative drainage as the independent variable to set cut-off values.

These results were similar to those described in previous reports $(13,14)$. Matsumoto et al. demonstrated that stapler use was associated with significantly shorter operative times and reduced rates of delayed air leakage compared to electrocautery (14). A prospective study comparing the use of a stapler and electrocautery, conducted by Chen $e t$ al., demonstrated higher postoperative complication rates in the electrocautery than stapler group (13). Based on these results, the use of a stapler can be considered superior in terms of the rates of postoperative complications, especially pulmonary fistula. Pulmonary segmentectomy is a minimally invasive procedure suitable for patients with compromised lung function, such as elderly patients. Minimizing complications is important in these patients to ensure rapid postoperative recovery. Prolonged air leak (PAL) occurs more frequently with the use of electrocautery than staples, and is associated with significant morbidity and an increased risk of pulmonary complications (19).

Several studies have reported that the remnant lung shrinks after the use of staplers, which leads to worse postoperative pulmonary function compared to electrocautery $(20,21)$. Asakura et al. reported that the use of staplers for division of the intersegmental plane of pig lungs reduced the volume of preserved lobes compared to the use of electrocautery (22). Conversely, Tao et al. found that the use of staplers did not negatively affect the volume of remnant lungs after segmentectomy compared to the use of electrocautery, based 
Table 3 Perioperative outcomes of the two groups

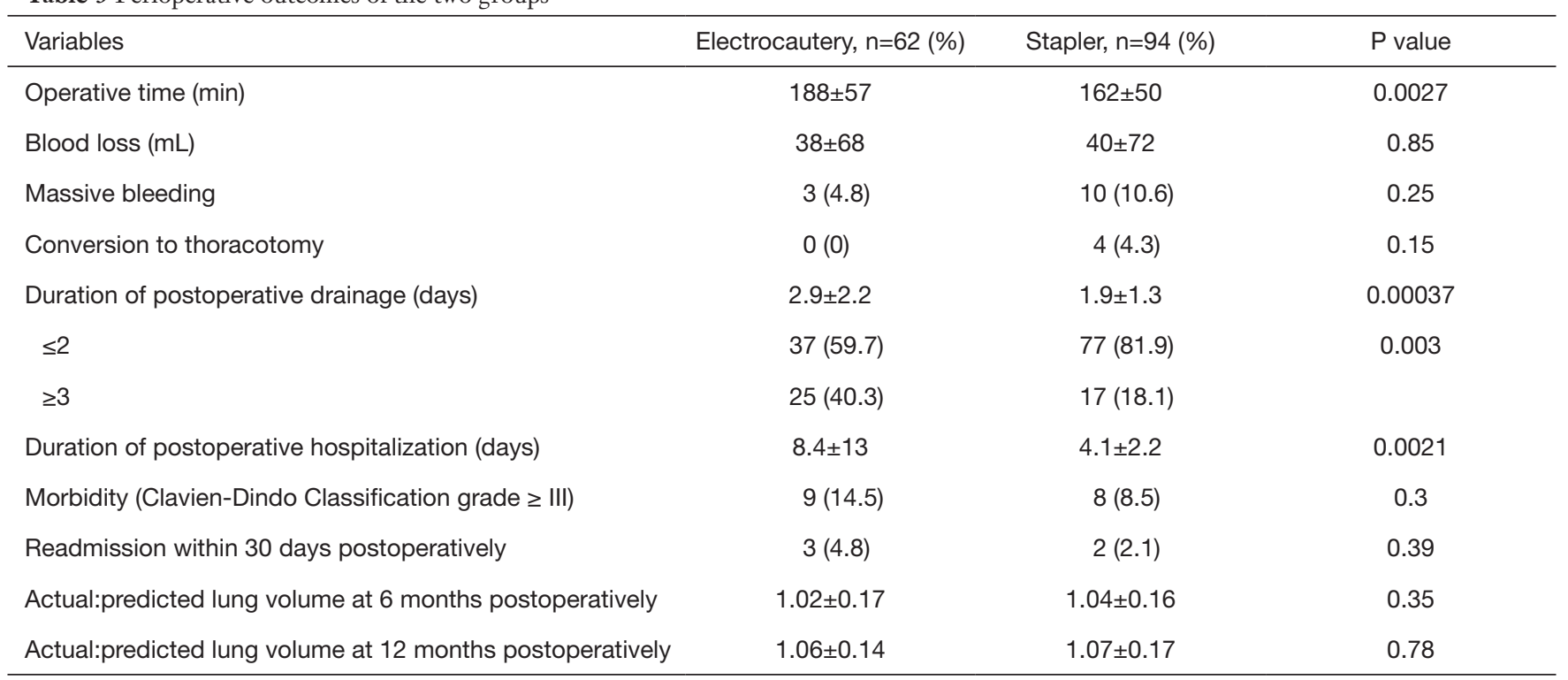
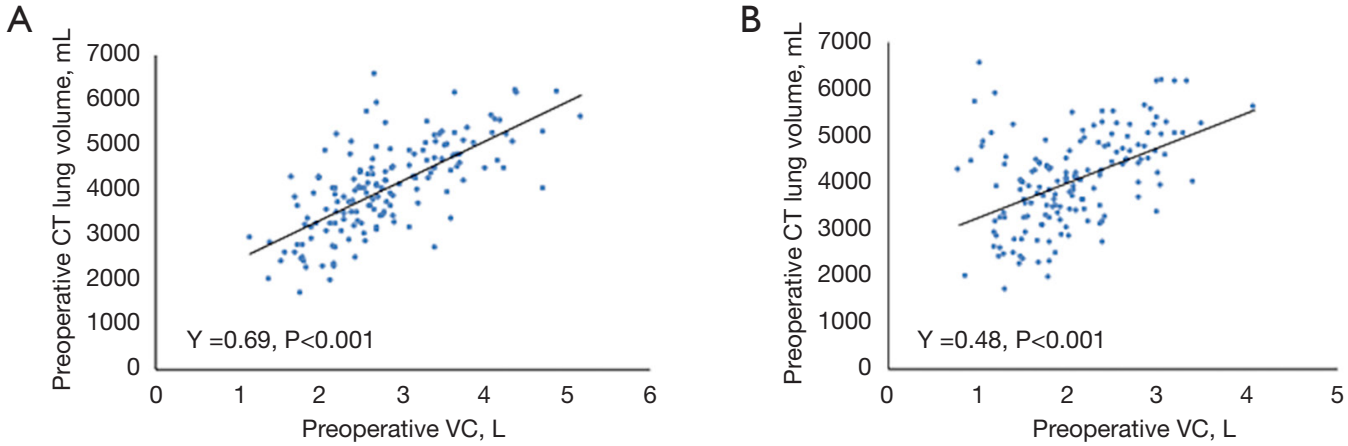

Figure 3 Preoperative lung volumes calculated by Ziostation2. VC, vital capacity.

Table 4 Multivariate analysis to identify the factors that contributed to the incidence of postoperative $\geq 3$ days drainage

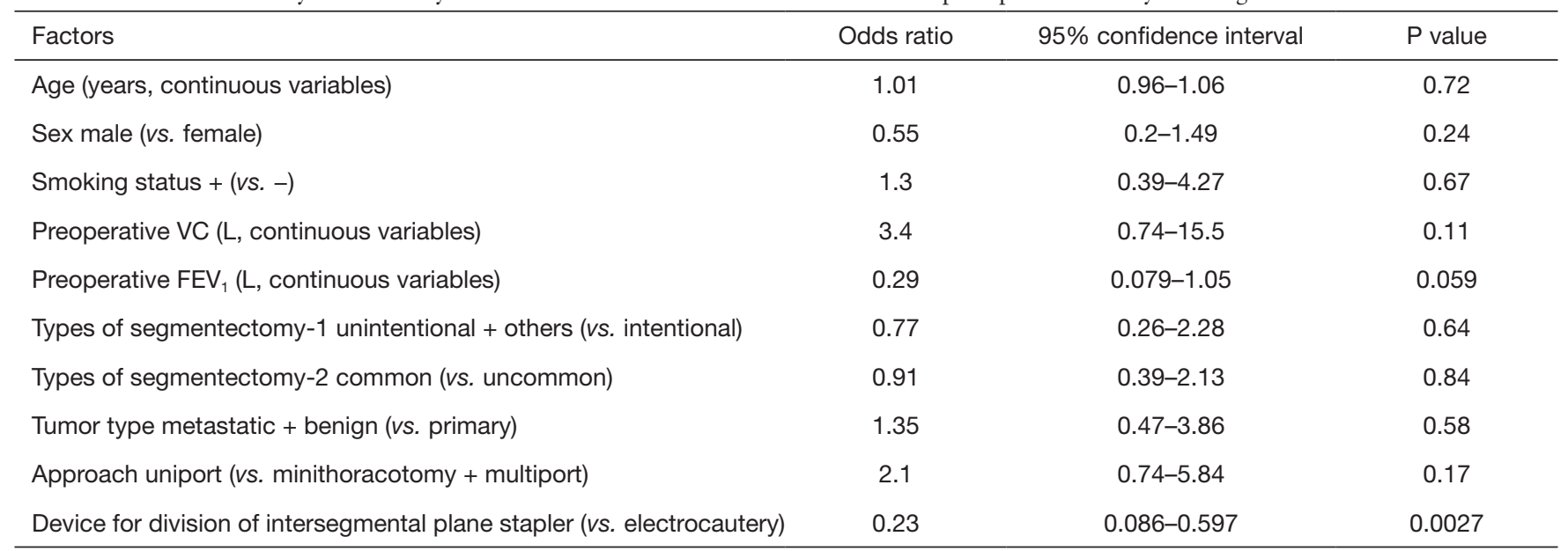

VC, vital capacity; $\mathrm{FEV}_{1}$, forced expiratory volume in 1 second. 
Table 5 Preoperative characteristics and perioperative outcomes of the electrocautery and stapler groups (common segmentectomy patients)

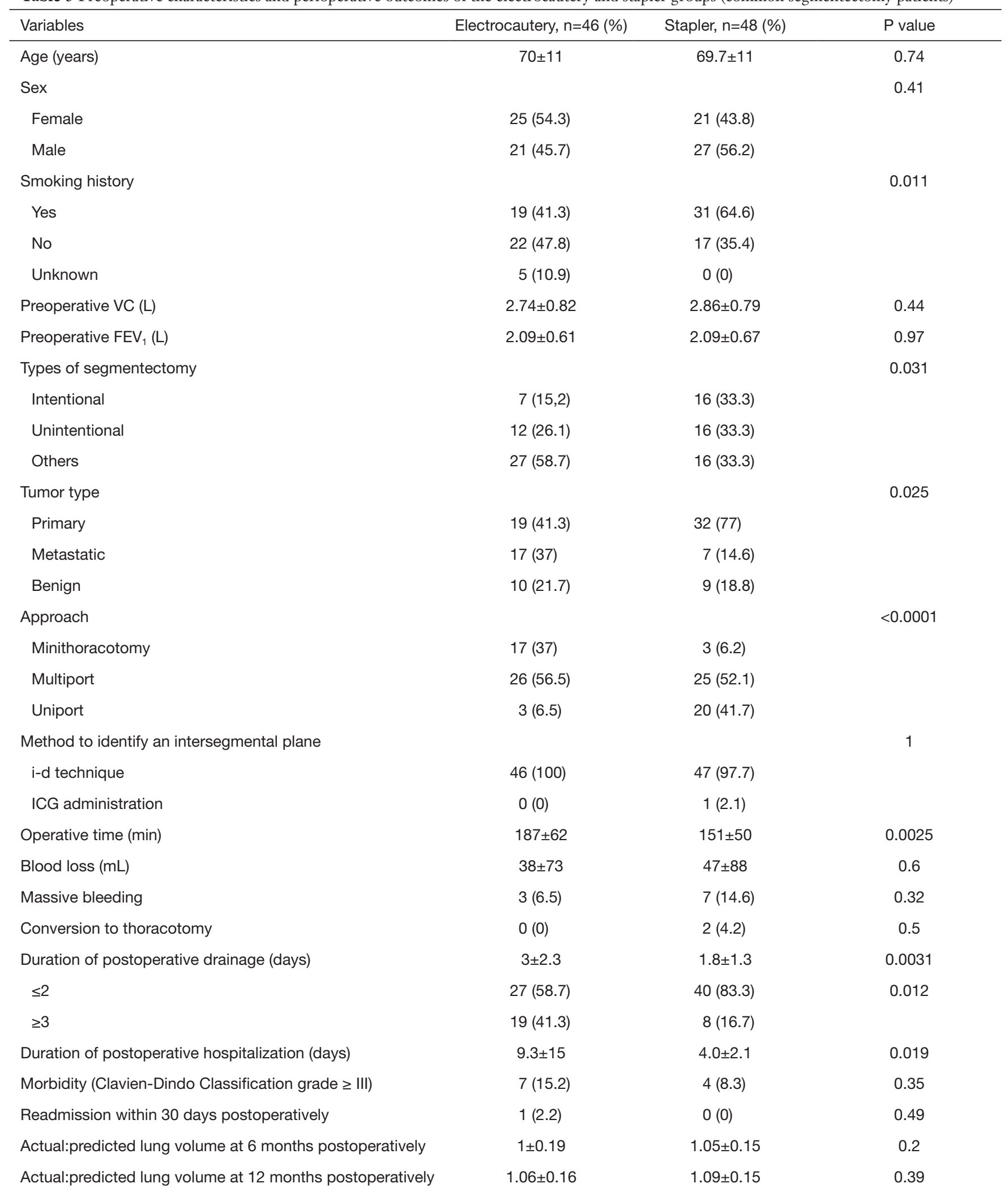

VC, vital capacity; $\mathrm{FEV}_{1}$, forced expiratory volume in 1 second; i-d technique, inflation-deflation technique; ICG, indocyanine green. 
Table 6 Preoperative characteristics and perioperative outcomes of the electrocautery and stapler groups (uncommon segmentectomy patients)

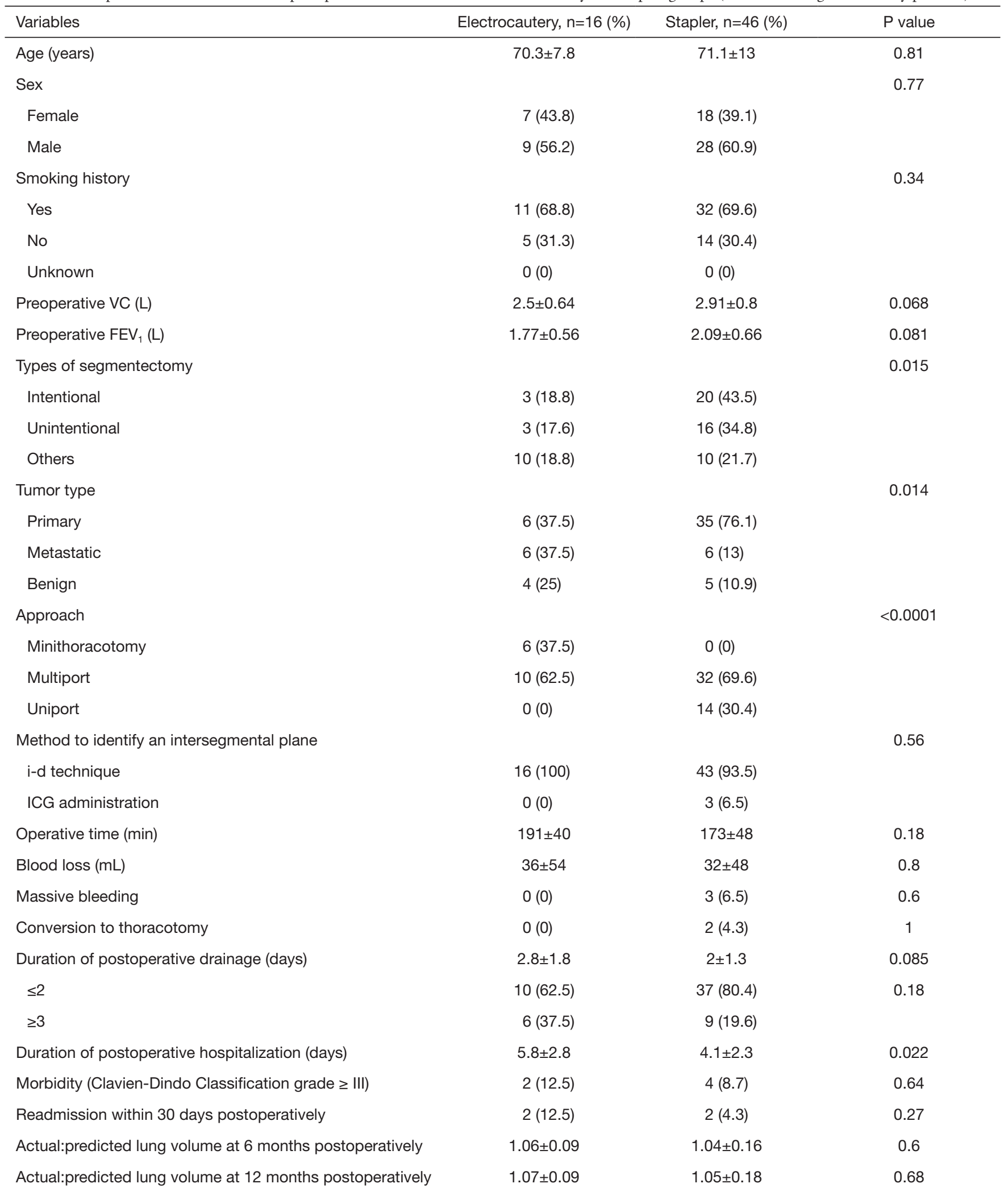

VC, vital capacity; FEV $_{1}$, forced expiratory volume in 1 second; i-d technique, inflation-deflation technique; ICG, indocyanine green. 
on a small number of participants (15). Therefore, the remnant lung can expand, and lung volumes can be preserved, even with use of a stapler. Prospective, comparative studies are required to confirm these results. However, such studies may be difficult to conduct because of the high frequency of postoperative complications in these patients (13).

Although the goal of lung volume preservation was to maintain lung function, data on postoperative pulmonary function were not collected in the present study. This is a retrospective study using clinical data that did not include postoperative pulmonary function that our team does not routinely examine postoperatively. Therefore, although the lung volumes were preserved, it is unclear if pulmonary functions were also preserved. However, our study revealed that preoperative lung volume was significantly correlated with preoperative pulmonary function. In addition, several authors previously reported that lung volumes calculated via quantitative $\mathrm{CT}$ analysis correlated well with the actual postoperative values, and were easier to calculate compared to other procedures such as ventilation/perfusion scintigraphy (23-26). In addition to preserved lung function, preserved lung volume is associated with improved outcomes in cases of repeat surgery (27).

In this study, an intersegmental plane was identified using either the conventional inflation-deflation technique or near-infrared imaging after ICG administration although the latter technique was applied only for 4 cases. Additionally, intersegmental pulmonary veins in the hilum were used as landmarks to identify the intersegmental plane when an intersegmental plane was not clearly identified using those techniques. These technical differences to identify an intersegmental plane might affect the postoperative lung volume because the demarcation line was a little different in each technique.

We performed subset analyses of perioperative outcomes and postoperative lung volumes in common and uncommon segmentectomies. An uncommon segmentectomy is technically more challenging because it requires division of multiple intersegmental surfaces, and exposure of the peripheral pulmonary vessels and bronchus. To overcome these technical difficulties, Suzuki et al. recommended that electrocautery should be used for division of the intersegmental plane in uncommon segmentectomy rather than staplers (3). However, in that study, electrocautery was associated with a higher incidence of PAL. Our study demonstrated that staplers and electrocautery had similar perioperative outcomes and postoperative lung volumes in uncommon segmentectomy. Although the number of patients who underwent uncommon segmentectomy in our study was small, the use of staplers resulted in satisfactory perioperative outcomes and postoperative lung expansion in these cases, without the need for electrocautery.

\section{Limitations}

This study had several limitations. First, it was a retrospective study with a small sample size and singleinstitution design. Second, the study period was relatively long. Third, the choice of device including electrocautery or staples for division of an intersegmental plane was significantly correlated with the surgical approach including minithoracotomy, multiport and uniport. Fourth, staples were more frequently used in the latter of study period because staples were usually chosen in the uniportal approach, which might affect better perioperative outcomes in the stapler group. Additionally, postoperative lung volume was predicted based on the number of resected segments, because Ziostation2 software could only examine the existing lung volume. Therefore, bias may have been introduced in patients with target segments larger or smaller than predicted. Moreover, no postoperative pulmonary function, which was the most important for postoperative patients' quality of life, was measured. Finally, we did not evaluate the cancer outcomes.

\section{Conclusions}

The use of staplers for division of the intersegmental plane during pulmonary segmentectomy led to better perioperative outcomes and similar postoperative remnant lung volumes compared to electrocautery. Moreover, even for the technically difficult uncommon type of segmentectomy, use of a stapler was not inferior to use of electrocautery about those evaluations. Therefore, we conclude that a stapler should be used for division of the intersegmental plane. Future studies should compare cancer outcomes between the use of staplers and electrocautery.

\section{Acknowledgments}

The authors thank the surgeons and their coworkers who performed the surgeries, and the editors and reviewers who provided assistance with the manuscript.

Funding: None. 


\section{Footnote}

Reporting Checklist: The authors have completed the STROBE reporting checklist. Available at https://dx.doi. org/10.21037/jtd-21-1397

Data Sharing Statement: Available at https://dx.doi. org/10.21037/jtd-21-1397

Peer Review File: Available at https://dx.doi.org/10.21037/ jtd-21-1397

Conflicts of Interest: All authors have completed the ICMJE uniform disclosure form (available at https://dx.doi. org/10.21037/jtd-21-1397). HI serves as an unpaid editorial board member of Fournal of Thoracic Disease from August 2020 to July 2022. The other authors have no conflicts of interest to declare.

Ethical Statement: The authors are accountable for all aspects of the work in ensuring that questions related to the accuracy or integrity of any part of the work are appropriately investigated and resolved. This study was conducted in accordance with the Declaration of Helsinki (2013 revision), and approved by the institutional ethics board of Japanese Red Cross Maebashi Hospital (No.: 2020-53, date: March 1, 2021). Informed consent was waived for this retrospective analysis.

Open Access Statement: This is an Open Access article distributed in accordance with the Creative Commons Attribution-NonCommercial-NoDerivs 4.0 International License (CC BY-NC-ND 4.0), which permits the noncommercial replication and distribution of the article with the strict proviso that no changes or edits are made and the original work is properly cited (including links to both the formal publication through the relevant DOI and the license). See: https://creativecommons.org/licenses/by-nc-nd/4.0/.

\section{References}

1. Vansteenkiste J, Crinò L, Dooms C, et al. 2nd ESMO Consensus Conference on Lung Cancer: early-stage nonsmall-cell lung cancer consensus on diagnosis, treatment and follow-up. Ann Oncol 2014;25:1462-74.

2. Detterbeck FC, Mazzone PJ, Naidich DP, et al. Screening for lung cancer: Diagnosis and management of lung cancer, 3rd ed: American College of Chest
Physicians evidence-based clinical practice guidelines. Chest 2013;143:e78S-92S.

3. Suzuki K, Saji H, Aokage K, et al. Comparison of pulmonary segmentectomy and lobectomy: Safety results of a randomized trial. J Thorac Cardiovasc Surg 2019;158:895-907.

4. Blades B. CONSERVATION OF LUNG TISSUE BY PARTIAL LOBECTOMY. Ann Surg 1943;118:353-65.

5. Okada M, Mimura T, Ikegaki J, et al. A novel videoassisted anatomic segmentectomy technique: selective segmental inflation via bronchofiberoptic jet followed by cautery cutting. J Thorac Cardiovasc Surg 2007;133:753-8.

6. Misaki N, Chang SS, Gotoh M, et al. A novel method for determining adjacent lung segments with infrared thoracoscopy. J Thorac Cardiovasc Surg 2009;138:613-8.

7. Oh S, Suzuki K, Miyasaka Y, et al. New technique for lung segmentectomy using indocyanine green injection. Ann Thorac Surg 2013;95:2188-90.

8. Sato M, Murayama T, Nakajima J. Techniques of staplerbased navigational thoracoscopic segmentectomy using virtual assisted lung mapping (VAL-MAP). J Thorac Dis 2016;8:S716-30.

9. Ojanguren A, Gossot D, Seguin-Givelet A. Division of the intersegmental plane during thoracoscopic segmentectomy: is stapling an issue? J Thorac Dis 2016;8:2158-64.

10. Pardolesi A, Park B, Petrella F, et al. Robotic anatomic segmentectomy of the lung: technical aspects and initial results. Ann Thorac Surg 2012;94:929-34.

11. Okada M, Mimura T, Ikegaki J, et al. A novel videoassisted anatomic segmentectomy technique: selective segmental inflation via bronchofiberoptic jet followed by cautery cutting. J Thorac Cardiovasc Surg 2007;133:753-8.

12. Oizumi H, Kanauchi N, Kato H, et al. Total thoracoscopic pulmonary segmentectomy. Eur J Cardiothorac Surg 2009;36:374-7; discussion 377.

13. Chen X, Jin R, Xiang J, et al. Methods for Dissecting Intersegmental Planes in Segmentectomy: A Randomized Controlled Trial. Ann Thorac Surg 2020;110:258-64.

14. Matsumoto M, Shirahashi K, Yamamoto H, et al. Division of the intersegmental plane using electrocautery for segmentectomy in clinical stage I non-small cell lung cancer. J Thorac Dis 2018;10:S1215-21.

15. Tao H, Tanaka T, Hayashi T, et al. Influence of stapling the intersegmental planes on lung volume and function after segmentectomy. Interact Cardiovasc Thorac Surg 2016;23:548-52. 
16. Tao H, Hayashi M, Furukawa M, et al. Influence of intersegmental plane size and segment division methods on preserved lung volume and function after pulmonary segmentectomy. Gen Thorac Cardiovasc Surg 2019;67:234-8.

17. Brunelli A. Uncommon pulmonary anatomic segmentectomies: state of the art and technical aspects. J Vis Surg 2018;4:175.

18. Bolliger CT, Wyser C, Roser H, et al. Lung scanning and exercise testing for the prediction of postoperative performance in lung resection candidates at increased risk for complications. Chest 1995;108:341-8.

19. Attaar A, Luketich JD, Schuchert MJ, et al. Prolonged Air Leak After Pulmonary Resection Increases Risk of Noncardiac Complications, Readmission, and Delayed Hospital Discharge: A Propensity Score-adjusted Analysis. Ann Surg 2021;273:163-72.

20. Nomori H, Shiraishi A, Cong Y, et al. Differences in postoperative changes in pulmonary functions following segmentectomy compared with lobectomy. Eur J Cardiothorac Surg 2018;53:640-7.

21. Okada M, Tsutani Y, Ikeda T, et al. Radical hybrid videoassisted thoracic segmentectomy: long-term results of minimally invasive anatomical sublobar resection for treating lung cancer. Interact Cardiovasc Thorac Surg 2012;14:5-11.

22. Asakura K, Izumi Y, Kohno M, et al. Effect of

Cite this article as: Yazawa T, Igai H, Numajiri K, Ohsawa F, Matsuura N, Kamiyoshihara M. Comparison of stapler and electrocautery for division of the intersegmental plane in lung segmentectomy. J Thorac Dis 2021;13(11):6331-6342. doi: $10.21037 /$ jtd-21-1397 cutting technique at the intersegmental plane during segmentectomy on expansion of the preserved segment: comparison between staplers and scissors in ex vivo pig lung. Eur J Cardiothorac Surg 2011;40:e34-8.

23. Ueda K, Tanaka T, Li TS, et al. Quantitative computed tomography for the prediction of pulmonary function after lung cancer surgery: a simple method using simulation software. Eur J Cardiothorac Surg 2009;35:414-8.

24. Ueda K, Tanaka T, Hayashi M, et al. Computed tomography-defined functional lung volume after segmentectomy versus lobectomy. Eur J Cardiothorac Surg 2010;37:1433-7.

25. Yoshimoto K, Nomori H, Mori T, et al. Quantification of the impact of segmentectomy on pulmonary function by perfusion single-photon-emission computed tomography and multidetector computed tomography. J Thorac Cardiovasc Surg 2009; 137:1200-5.

26. Yoshimoto K, Nomori H, Mori T, et al. Prediction of pulmonary function after lung lobectomy by subsegments counting, computed tomography, single photon emission computed tomography and computed tomography: a comparative study. Eur J Cardiothorac Surg 2009;3 5:408-13.

27. Igai H, Kamiyoshihara M, Kawatani N, et al. A case report: right upper lobectomy with middle lobe preservation after right lower lobectomy. Surg Case Rep 2015;1:19. 